\title{
Automatic Detection and Recognition of Lung Abnormalities in Helical CT Images Using Deformable Templates
}

\author{
Aly Farag ${ }^{1}$, Ayman El-Baz ${ }^{1}$, Georgy G. Gimel'farb² ${ }^{2}$, Robert Falk ${ }^{3}$, and \\ Stephen G. Hushek ${ }^{4}$ \\ 1 Computer Vision and Image Processing Laboratory \\ University of Louisville, Louisville, KY 40292, USA. \\ \{farag, elbaz\}@cvip.Louisville.edu, http://www.cvip.louisville.edu \\ 2 Department of Computer Science, Tamaki Campus \\ University of Auckland, Auckland, New Zealand. \\ 3 Director, Medical Imaging Division, Jewish Hospital, Louisville, KY, USA. \\ 4 Technical Director, iMRI Department, Norton Hospital, Louisville, KY, USA.
}

\begin{abstract}
Automatic detection of lung nodules is an important problem in computer analysis of chest radiographs. In this paper we propose a novel algorithm for isolating lung nodules from spiral CT scans. The proposed algorithm is based on using four different types of deformable templates describing typical geometry and gray level distribution of lung nodules. These four types are $(i)$ solid spherical model of large-size calcified and non-calcified nodules appearing in several successive slices; $(i i)$ hollow spherical model of large lung cavity nodules; (iii) circular model of small nodules appearing in only a single slice; and $(i v)$ semicircular model of lung wall nodules. Each template has a specific gray level pattern which is analytically estimated in order to fit the available empirical data. The detection combines the normalized cross-correlation template matching by genetic optimization and Bayesian post-classification. This approach allows for isolating abnormalities which spread over several adjacent CT slices. Experiments with 200 patients' CT scans show that the developed techniques detect lung nodules more accurately than other known algorithms.
\end{abstract}

\section{Introduction}

Automatic detection of lung nodules is an important problem in computer analysis of chest radiographs. One in every 18 women and every 12 men develop lung cancer, making it the leading cause of cancer deaths. Early detection of lung tumors (visible on the chest film as nodules) may increase the patient's chance of survival [2].

At present, low-dose spiral computed tomography (LDCT) is of prime interest for screening (high risk) groups for early detection of lung cancer 2]. The LDCT provides chest scans with very high spatial, temporal, and contrast resolution of anatomic structures and is able to gather a complete 3D volume of a human thorax in a single breath-hold [3]. The automatic screening typically involves 
two-stage detection of lung abnormalities (nodules). First, the initial candidate nodules are selected and then the false candidates, called false positive nodules (FPNs) are partially eliminated while preserving the true ones (TPNs).

At the first stage, conformal nodule filtering [4] or unsharp masking [5] can enhance nodules and suppress other structures to separate the candidates from the background by simple thresholding (to improve the separation, background trend is corrected in 6] within image regions of interest). Circular nodule candidates can be detected by template matching [5] or Hough transform [7]. Other methods detect lung nodules by using morphological operators such as the algorithm proposed in 8]. The drawbacks of this algorithm are it fails to detect cavity lung nodules and has difficulties in detecting lung wall nodules.

The FPNs are excluded at the second stage by feature extraction and classification 6 9]. Such features as circularity, size, contrast [6], or local curvature 9] are extracted by morphological techniques, and artificial neural networks (ANN) are frequently used as post-classifiers [10]. The critical issue is to adequately discriminate between the nodules and non-nodules.

In this paper nodule types are modelled with four central-symmetric deformable templates: $(i)$ solid spherical model of large-size (above $10 \mathrm{~mm}$ ) calcified and non-calcified nodules appearing in several successive slices; (ii) hollow spherical model of large lung cavity nodules; ( $i i i$ ) circular model of small nodules appearing in only a single slice; and ( $i v)$ semicircular model of lung wall nodules. This approach allows for isolating abnormalities which spread over several adjacent CT slices.

Each template has a specific gray level pattern which is analytically estimated in order to fit the available empirical data. Normalized cross-correlation is used for template matching. The 3D or 2D position, size, and gray level pattern of each template is adjusted to the most similar part of the segmented veins, arteries, and lung abnormalities by a genetic optimization technique [11. After all the candidates are detected, a supervised Bayesian classification of geometric and textural features of the candidate nodules partially excludes the FPNs.

\section{Deformable Templates of Abnormalities}

Our detection of lung nodules begins with two segmentation stages which considerably reduce the search space. At the first stage shown in Fig.11(a) and Fig.11(b), lung tissues are separated from the surrounding anatomical structures, e.g., ribs, liver, and other organs, appearing in the chest CT scans. The second stage extracts arteries, veins, bronchi, and lung abnormalities (see Fig. 11(c)) from the already segmented lung tissues. Segmentation algorithms are based on representing each CT slice as a sample of a Markov-Gibbs random field of region labels and gray levels. Details of the algorithms are presented in [1, and in this paper we focus only on the third stage of detecting and classifying the nodules among the extracted objects. Figure 2(a) shows the empirical gray level distribution over the extracted regions in Fig. 1(c). Both the nodules and normal tissues such as arteries, veins, and bronchi, have almost the same gray level distribu- 
tions, so abnormality detection must include their geometrical shape. Four basic classes of lung abnormalities are: small calcified; large calcified; non-calcified; and cavity nodules. The first three classes tend to have solid spherical shapes, whereas the cavity nodules are hollow spheres. Generally, the smaller nodules

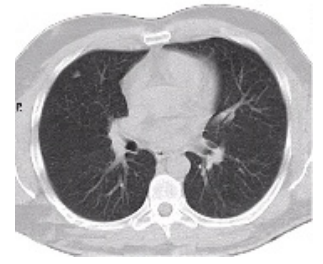

(a)

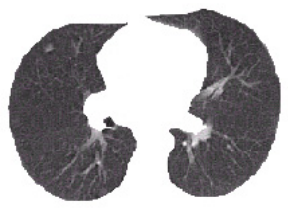

(b)

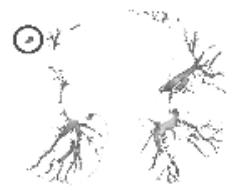

(c)

Fig. 1. First two segmentation steps.

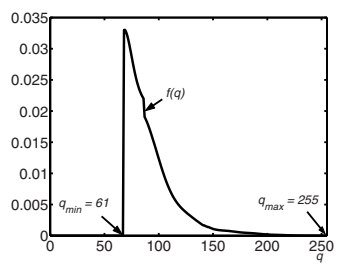

(a)

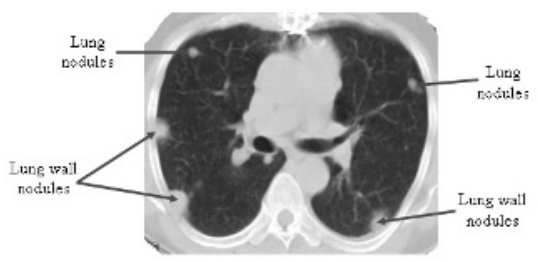

(b)

Fig. 2. (a) The empirical gray level distribution over the extracted regions in Fig. 1(c) (b) Nodule positions and shapes.

appear only in a single 2D slice like in Fig. 2(b), whereas the larger ones spread over a $3 \mathrm{D}$ volume represented by several successive slices. The lung wall nodules may also appear in one or more slices, depending on their size. However, they are semicircular in shape as shown in Fig. 2(b). Our analysis of 2D CT slices suggests that spatial changes of gray levels across the central cross-section of a solid-shape 3D nodule or across a solid-shape 2D nodule can be approximated with a central-symmetric Gaussian-like template $q(r)=q_{\max } \exp \left(-(r / \rho)^{2}\right)$; $0 \leq r \leq R$. Here, $r$ is the radius from the template's center and $q(r)$ is the gray level in a template point with Cartesian coordinates $(\xi, \eta)$ with respect to the center (i.e., $r^{2}=\xi^{2}+\eta^{2}$ ), $q_{\max }$ denotes the maximum gray level for the template, $R$ is the template radius depending on the minimum gray level $q_{\min }=q(R)$, and the parameter $\rho$ specifies how fast the signals decrease across the template. 


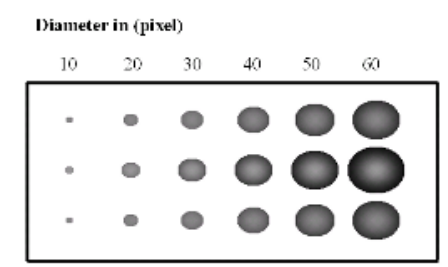

(a)

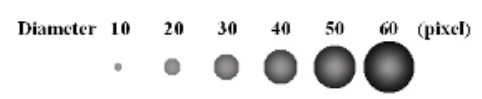

(c)

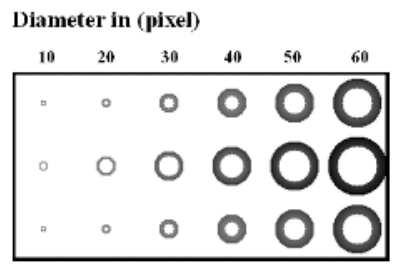

(b)

Diameter 20 pixels

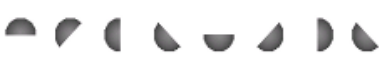

(d)

Fig. 3. Examples of the deformed templates for GA template matching process. (a) Solid spherical models consisting of three slices to detect large calcified and non-calcified nodules. (b) Hollow spherical models consisting of three slices to detect thick cavity nodules. (c) Circular models to detect small nodules. (d) Semicircular models to detect lung wall nodules.

\section{Genetic Algorithm(GA) Template Matching}

GA template matching is used to effectively search for the location of lung nodules scattered within the lung areas. In this method, the genetic algorithm is used to determine the target position in an observed image and to select a suitable radius to generate a template model for the template matching process. Details of the GA process are described below.

\subsection{Template Identification}

The CT slices in our study have in-plane spatial resolution of $0.4 \mathrm{~mm}$ per pixel so that the radius range for all lung nodules is $R=5-30$ pixels. Because the third spatial axis has lower resolution, for large solid and hollow lung nodules we use the 3-layer template. Thin lung nodules appearing only in a single slice have the circular templates. The lung wall nodules are of semicircular shape. We assume that the template deformations, other than translations are restricted to different scales (radii) of all the templates and also different (orientation) angles of the semicircular templates. Examples of the deformed templates are presented in Fig. 3. In order to get better matching between the template model and the lung nodules we have to generate a template which has a density close to the density of the segmented veins, arteries, and lung abnormalities which are shown in Fig. 2(a). Gray level distribution density over the 2D Gaussian template can be found as follows: 


$$
\psi(q)=2 \pi r(q)
$$

since $r(q)=\rho \sqrt{\ln q_{\max }-\ln q}$, then $\psi(q)$ can be expressed as follows:

$$
\psi(q)=2 \pi \rho \sqrt{l n q_{\max }-\ln q}
$$

In order to compute the density for the template using Eq.(2), we need to estimate the parameter $\rho$. For a template which has radius $R$, the parameter $\rho$ can be estimated from the following equation:

$$
\rho=R\left(\ln q_{\max }-\ln q_{\min }\right)^{-\frac{1}{2}}
$$

By using Eq.(3), the gray level distribution density over the 2D Gaussian template can be expressed in the following closed form:

$$
\psi\left(q \mid q_{\min }, q_{\max }\right)=2 \pi R \sqrt{\frac{\ln q_{\max }-\ln q}{\ln q_{\max }-\ln q_{\min }}}
$$

This relationship allows us to roughly estimate the template parameters $q_{\max }$ and $q_{\min }$ from the empirical density in Fig. 2(2) (a) (in this particular case $q_{\max }=$ 255 and $q_{\min }=61$ ). In particular, for the circular templates of the radii $R=5$ and 30, the estimated $\rho=4.18$ and 25.08, respectively. Figure 4 demonstrates how close the empirical gray level distribution for the objects in Fig. 1 1 c) are to the estimated distribution for the above two templates under its discretization.

In the case of the $3 \mathrm{D}$ solid spherical templates, the $2 \mathrm{D}$ template is first identified for the central cross-section. Then the upper and lower cross-sections are specified by the same parameters in the following equation $\left(q_{t}(r)=\right.$ $\left.q_{\max } \exp \left(-\left(r^{2}+t^{2}\right) / \rho^{2}\right)\right)$ where $t$ is the slice thickness in pixels $(t=7$ in our experiments below). The radius of upper and lower circles is specified by the relationship $q_{t}(R)=q_{\min }$.

The hollow spherical templates to detect cavity lung nodules are obtained in a similar way by removing the central part of the solid templates up to $75 \%$ of the radius $R$.

\subsection{The GA Template Matching Process}

As mentioned above GA is used to determine the target position in an observed image and select a suitable radius to generate a template model. In this paper we use the genetic algorithm with the following structure (for more details about GA see 11).

- Chromosome: Each chromosome has 28 bits, of which 23 determine the target position. The 23 position bits are divided into 9-, 9-, and 5-bit sets corresponding to the coordinates $(x, y, z)$ respectively. The last 5 bits determine the radius of the generated templates $R$. Once we know $R, q_{\min }$, and $q_{\max }$ we calculate $\rho$ from Eq. 3 By using $\rho$, and $q_{\max }$ we generate the corresponding template. Then similarities between the cut image and the generated template are calculated. 

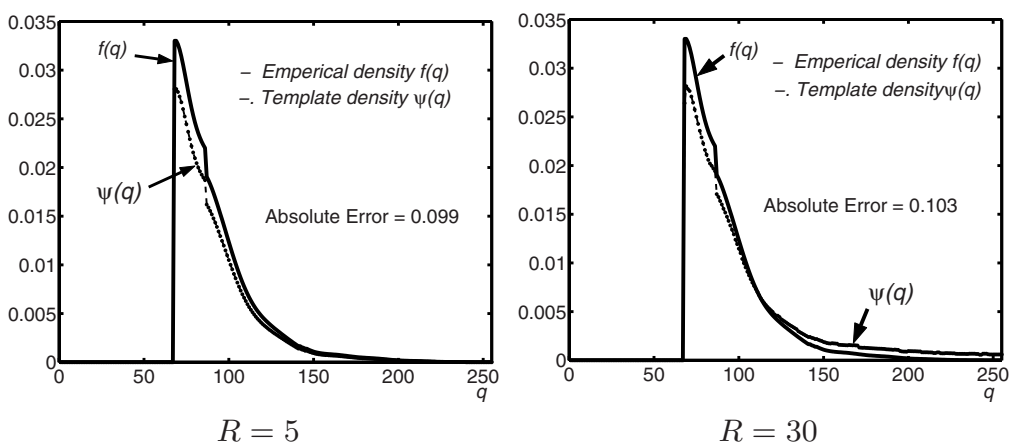

Fig. 4. Estimated template gray level distributions $(\psi(q))$ w.r.t. the empirical density $(f(q))$.

- Fitness: We define the fitness of an individual template as the "similarity" calculated by the normalized cross-correlation of two images $a$, and $b[12$, as

$$
\text { Similarity }_{a, b}=\frac{\sum_{i=1}^{n} \sum_{j=1}^{n}\left(a_{i j}-m_{a}\right)\left(b_{i j}-m_{b}\right)}{\sqrt{\sum_{i=1}^{n} \sum_{j=1}^{n}\left(a_{i j}-m_{a}\right)^{2}} \sqrt{\sum_{i=1}^{n} \sum_{j=1}^{n}\left(b_{i j}-m_{b}\right)^{2}}}
$$

where $m_{a}=\frac{1}{n} \sum_{i=1}^{n} \sum_{j=1}^{n} a_{i j}, m_{b}=\frac{1}{n} \sum_{i=1}^{n} \sum_{j=1}^{n} b_{i j}$, the values $a$ and $b$ signify the images for comparison. The $a_{i j}$ is the value of a pixel at site $(i, j)$ in image $a$, similarly $b_{i j}$.

The matching algorithm runs separately for each type of lung abnormality (Note that for semicircular template model we add another part in the chromosome that represent the angle). All spatial locations where the similarity score is greater than a certain threshold (in our experiments 0.8) are extracted as candidate nodules.

\section{Post-classification of Nodule Features}

Because actual lung nodules are not exactly spherical, circular, or semicircular, some true nodules can be missed. A number of false positive nodules (FPNs) can also be encountered during the initial extraction of the candidates. To reduce the error rate, post-classification of the candidate nodules is performed with three textural and geometric features of each detected nodule: ( $i$ ) radial non-uniformity $U=\max _{\theta}(d(\theta))-\min _{\theta}(d(\theta))$ of its borders (here, $d(\theta)$ is the distance at the angle $\theta$ between the center of the template and the border of the segmented object in Fig. 1(c)); (ii) mean gray level $\left(q_{\text {ave }}\right)$ over the 3D or 2D nodular template, and (iii) the 10\%-tile gray level for the marginal gray level distribution over the $3 \mathrm{D}$ or $2 \mathrm{D}$ nodular template. To distinguish between the FPNs and true positive nodules(TPNs), we use Bayesian supervised classifier learning statistical 
characteristics of these features from a training set of false and true nodules. All three features (i)-(iii) are used to classify the FPNs in lung, while only the last two features can be applied to the lung wall nodules.

\section{Experimental Results and Conclusions}

The algorithm was tested on the CT scans of 200 subjects enrolled to the screening study. Among them, 21 subjects had abnormalities in their CT scans and 179 subjects were normal (this classification validated by a radiologist). At stage one, the template matching extracted 110 true candidates (out of the true 130 nodules) and 49 FPNs.

The classification at stage two reduced the number of the FPNs to 12 but simultaneously rejected three true nodules. Thus the final number of the TPNs became 107 out of 130 giving the overall correct detection rate of $82.3 \%$ with the FPNs rate of $9.2 \%$. Table 1 presents the numbers of TPNs and FPNs before and after the post-classification stage.

Table 1. Detection rate for different types of abnormalities (TPNs : the nodules determined by a radiologist).

\begin{tabular}{|c|c|c|c|c|}
\hline $\begin{array}{c}\text { Type of Lung } \\
\text { Nodules }\end{array}$ & $\begin{array}{c}\text { True detecting } \\
\text { nodules before } \\
\text { removing FPNs }\end{array}$ & $\begin{array}{c}\text { False detecting } \\
\text { nodules before } \\
\text { removing FPNs }\end{array}$ & $\begin{array}{c}\text { True detecting } \\
\text { nodules after } \\
\text { removing FPNs }\end{array}$ & $\begin{array}{c}\text { False detecting } \\
\text { nodules after } \\
\text { removing FPNs }\end{array}$ \\
\hline Lung wall & $28: 29$ & 8 & $27: 29$ & 2 \\
\hline Calcified & $46: 49$ & 4 & $46: 49$ & 1 \\
\hline Non-calcified & $12: 18$ & 5 & $12: 18$ & 3 \\
\hline Cavity & $8: 11$ & 7 & $8: 11$ & 1 \\
\hline Small & $17: 23$ & 25 & $15: 23$ & 5 \\
\hline
\end{tabular}

Table 2. Detection rate for different types of abnormalities by using the algorithm proposed in [12] (TPNs: the nodules determined by a radiologist).

\begin{tabular}{|c|c|c|c|c|}
\hline $\begin{array}{c}\text { Type of Lung } \\
\text { Nodules }\end{array}$ & $\begin{array}{c}\text { True detecting } \\
\text { nodules before } \\
\text { removing FPNs }\end{array}$ & $\begin{array}{c}\text { False detecting } \\
\text { nodules before } \\
\text { removing FPNs }\end{array}$ & $\begin{array}{c}\text { True detecting } \\
\text { nodules after } \\
\text { removing FPNs }\end{array}$ & $\begin{array}{c}\text { False detecting } \\
\text { nodules after } \\
\text { removing FPNs }\end{array}$ \\
\hline Lung wall & $14: 29$ & 86 & $13: 29$ & 17 \\
\hline Calcified & $31: 49$ & 35 & $31: 49$ & 9 \\
\hline Non-calcified & $14: 18$ & 25 & $14: 18$ & 14 \\
\hline Cavity & - & - & - & - \\
\hline Small & $10: 23$ & 34 & $9: 23$ & 12 \\
\hline
\end{tabular}


To illustrate the efficiency of the proposed algorithm we compare the results obtained by the proposed algorithm with the results obtained by other algorithms. To the best of our knowledge, there is only one related work [12] that detects lung nodules from spiral CT scan by using a template matching method. The proposed algorithm by Lee [12] detects only three types of nodules: large lung nodules, small lung nodules, and lung wall nodules by using fixed templates and the parameters for each template are selected manually from the given data set. We ran Lee's algorithm on the same data sets. The algorithm detects at stage one, 69 true candidates (out of the true 130 nodules) and 180 FPNs. The classification at stage two reduced the number of FPNs to 52 but simultaneously rejected two true nodules. Thus the final number of TPNs became 67 out of 130 giving the overall correct detection rate of $51.5 \%$ with the FPNs rate of $40 \%$. Table 2 presents the details of the results obtained by the algorithm proposed in 12 . It is clear from Table 2 that this algorithm fails to detect large numbers of true nodules because this algorithm used fixed size templates and these templates sometimes give low correlation between the template and the true nodules. They estimate the parameters that determine the gray levels manually, and they use them for the whole volume. At times this is not the best estimation because the distribution for the gray level can change from one slice to another (depends on the cross section that scanned it, and the organs that appear in that cross section). In our proposed algorithm we estimate these parameters analytically using Eq. (3) for each CT slice.

Our experiments show that the proposed adaptive deformable templates, with analytical parameter estimation, allow for detection of more than $80 \%$ of the true lung abnormalities. The number of simultaneously detected false nodules can be considerably reduced by accounting for simple geometrical and textural features of the candidate nodules.

Acknowledgement. This research has been supported by grants from the Jewish Hospital Foundation and the Kentucky Lung Cancer Program.

\section{References}

1. Aly A. Farag, Ayman El-Baz, G.L.Gimel'farb, "Precise Image Segmentation by Iterative EM-Based Approxiation of Empirical Grey Level Distributions with Linear Combinations of Gaussions", IEEE Workshop on Learning in Computer Vision and Pattern Recognition, Washington, DC, June, 2004

2. P. M. Boiselle and C. S. White (Eds.) New Techniques in Thoracic Imaging. M. Dekker, New York, 2002.

3. L. Quekel, A.Kessels, R. Goei, and J. V. Engelshoven, "Miss rate of lung cancer on the chest radiograph in clinical practice," Chest, Vol. 115, no. 3, pp. 720-724, 1999.

4. S.-C. B. Lo, M. T. Freedman, J.-S. Lin, and S. K. Mun, "Automatic lung nodule detection using profile matching and back-propagation neural network techniques," J. Digital Imaging, Vol. 6, no. 1, pp. 48-54, 1993. 
5. F. Mao, W. Qian, J. Gaviria, and L. Clarke, "Fragmentary window filtering for multiscale lung nodule detection," Academic Radiology, Vol. 5, no. 4, pp. 306-311, 1998.

6. X. Xu, S. Katsuragawa, K. Ashizawa,H. MacMahon, and K. Doi, "Analysis of image features of histograms of edge gradient for false positive reduction in lung nodule detection in chest radiographs," Proc. SPIE, Vol. 3338, pp. 318-326, 1998.

7. W. Lampeter, "ANDS-V1 computer detection of lung nodules," Proc. SPIE, Vol. 555, pp. 253-261, 1985.

8. Catalin I. Fetita, Francoise Preteux, Catherine Beigelman-Aubry, and Philippe Grenier, "3D Automated Lung Nodule segmentation in HRCT," Proc. MICCAI, LNCS 2878, pp. 626-634, November 2003.

9. M. J. Carreira, D. Cabello, M. G. Penedo,and J. M. Pardo, "Computer aided lung nodule detection in chest radiography," in R. T. Chin et al. (Eds.) Image Analysis Applications and Computer Graphics, (Lecture Notes in Computer Science 1024), Springer, Berlin, pp. 331-338, 1995.

10. S-C. B. Lo, S.-L. A. Lou, J.-S. Lin, M. T. Freedman, M. V. Chien, and S. K. Mun, "Artificial convolution neural network techniques and applications for lung nodule detection," IEEE Trans. Med. Imaging, Vol. 14, pp. 711-718, August 1995.

11. D. E. Goldberg, Genetic Algorithms in Search, Optimization and Machine Learning. Addison-Wesley, Reading, Mass., 1989.

12. Y. Lee, T. Hara, H. Fujita, S. Itoh, and T. Ishigaki, "Automated Detection of Pulmonary Nodules in Helical CT Images Based on an Improved Template-Matching Technique," IEEE Tran. Med. Imaging, Vol. 20, pp. 595-604, July 2001. 